\title{
FUZZY STRICT PREFERENCE RELATIONS COMPATIBLE WITH FUZZY ORDERINGS
}

\author{
BONIFACIO LLAMAZARES \\ Department of Applied Economics, Valladolid University \\ Avda. Valle de Esgueva 6, E-47011 Valladolid, Spain \\ boni@eco.uva.es \\ BERNARD DE BAETS \\ Department of Applied Mathematics, Biometrics and Process Control \\ Ghent University, Coupure links 653, B-9000 Ghent, Belgium \\ bernard.debaets@ugent.be \\ Received (received date) \\ Revised (revised date)
}

\begin{abstract}
One of the most important issues in the field of fuzzy preference modelling is the construction of a fuzzy strict preference relation and a fuzzy indifference relation from a fuzzy weak preference relation. Here, we focus on a particular class of fuzzy weak preference relations, the so-called fuzzy orderings. The definition of a fuzzy ordering involves a fuzzy equivalence relation and, in this paper, the latter will be considered as the corresponding fuzzy indifference relation. We search for fuzzy strict preference relations compatible with a given fuzzy ordering and its fuzzy indifference relation. In many situations, depending on the t-norm and t-conorm used, this quest results in a unique fuzzy strict preference relation. Our aim is to characterize these fuzzy strict preference relations and to study their transitivity.
\end{abstract}

Keywords: Fuzzy ordering; fuzzy strict preference relation; transitivity.

\section{Introduction}

In the classical theory of preference modelling (see, for instance, Roubens and Vincke $^{1}$ ), it is usual to represent the agents' preferences on a set of alternatives $A$ by means of a reflexive relation $R$, called weak preference relation, where $a R b$ is interpreted as " $a$ is at least as good as $b$ ". From this relation, it is possible to obtain a strict preference relation $P$ (the asymmetric part of $R$ ) and an indifference relation $I$ (the symmetric part of $R$ ). The relation $P$ is used to denote strict preference, i.e., $a P b$ means that " $a$ is better than $b$ ". The relation $I$ is used to denote indistinguishability, i.e., $a I b$ means that " $a$ and $b$ are indifferent". These relations can be expressed as $P=R \cap\left(R^{t}\right)^{c}$ and $I=R \cap R^{t}$. Moreover, $P$ and $I$ satisfy the following properties: $P$ is asymmetric, i.e., $P \cap P^{t}=\emptyset ; I$ is symmetric; $P \cap I=\emptyset$ and $R=P \cup I$. 
A particular class of weak preference relations is the class of partial order relations, i.e., reflexive, antisymmetric and transitive relations. For such a partial order relation $R, I$ is the minimal reflexive relation, i.e. $I=\{(a, a) \mid a \in A\}$, and $P=R \backslash I$. Furthermore, $P$ is transitive and $I$ is an equivalence relation.

In fuzzy set theory, there are usually many options to generalize a given crisp concept or property. In particular, a fuzzy strict preference relation can be constructed from a given fuzzy weak preference relation in a multitude of ways (see, among others, De Baets and Van de Walle ${ }^{2}$, Perny and Roubens ${ }^{3}$, Van de Walle et al. ${ }^{4,6}$, Bufardi ${ }^{7,8}$, Llamazares ${ }^{9}$, De Baets and Fodor ${ }^{10}$ and Fono et al. $\left.{ }^{11}\right)$. Here, we study the fuzzy strict preference relations compatible with a given fuzzy partial order relation. To that end, we adopt the definition of a fuzzy partial order relation proposed by Höhle and Blanchard ${ }^{12}$, also called fuzzy ordering. Depending on the t-norm and t-conorm used for modelling intersection and union, we identify all possible fuzzy strict preference relations compatible with a given fuzzy ordering. In case there is a unique such relation, we provide a characterization.

Our paper is organized as follows. In Section 2 we introduce the notations used and some basic definitions. Section 3 contains the main results of the paper. Finally, some concluding remarks are pointed out in Section 4.

\section{Preliminaries}

Let $A$ be a set of alternatives with $|A| \geq 2$. A binary relation $Q$ on $A$ is a subset of $A^{2}=A \times A$. As all relations in this paper are binary, we will drop the term 'binary'. We will use $a Q b$ to denote $(a, b) \in Q$. The relation $\Delta$ is defined as $\Delta=\{(a, a) \mid a \in$ $A$ \}. Given a relation $Q$ on $A$, the relations $Q^{t}$ and $Q^{c}$ are defined as

$$
\begin{aligned}
& Q^{t}=\left\{(a, b) \in A^{2} \mid(b, a) \in Q\right\}, \\
& Q^{c}=\left\{(a, b) \in A^{2} \mid(a, b) \notin Q\right\} .
\end{aligned}
$$

A relation $Q$ on $A$ is called:

(i) reflexive if $\Delta \subseteq Q$;

(ii) symmetric if $Q \subseteq Q^{t}$;

(iii) asymmetric if $Q \cap Q^{t}=\emptyset$;

(iv) antisymmetric if $Q \cap Q^{t} \subseteq \Delta$;

(v) transitive if $(a Q b$ and $b Q c) \Rightarrow a Q c$ for all $a, b, c \in A$;

(vi) complete if $Q \cup Q^{t}=A^{2}$.

A relation is called an equivalence relation if it is reflexive, symmetric and transitive; it is called a partial order relation if it is reflexive, antisymmetric and transitive. A relation $R$ on $A$ is called a weak preference relation if it is reflexive. Any weak preference relation can be factorized in a strict preference relation, $P=R \cap\left(R^{t}\right)^{c}$, and an indifference relation, $I=R \cap R^{t}$. Moreover, the relations $P$ and $I$ are characterized through the following properties: $P$ is asymmetric, $I$ is symmetric, $P \cap I=\emptyset$ and $R=P \cup I$. 
A fuzzy subset $B$ of $A$ is a function $B: A \rightarrow[0,1]$, where $B(a)$ is the degree of membership of $a$ in $B$. The intersection and union of fuzzy sets are defined by means of triangular norms and conorms, respectively. A function $T:[0,1]^{2} \rightarrow[0,1]$ is a triangular norm (t-norm) if it is increasing, commutative, associative and has neutral element 1 ; it has absorbing element 0 . A function $S:[0,1]^{2} \rightarrow[0,1]$ is a triangular conorm (t-conorm) if it is increasing, commutative, associative and has neutral element 0 ; it has absorbing element 1.

Given a t-norm $T$ and a t-conorm $S$, the intersection and the union of two fuzzy subsets $B, C$ of $A$ are defined by

(i) $B \cap_{T} C(a)=T(B(a), C(a))$, for all $a \in A$;

(ii) $B \cup_{S} C(a)=S(B(a), C(a))$, for all $a \in A$.

The most important t-norms and t-conorms are listed next:

(i) $T_{\mathbf{M}}(x, y)=\min (x, y)$ and $S_{\mathbf{M}}(x, y)=\max (x, y)$;

(ii) $T_{\mathbf{P}}(x, y)=x y$ and $S_{\mathbf{P}}(x, y)=x+y-x y$;

(iii) $T_{\mathbf{L}}(x, y)=\max (x+y-1,0)$ and $S_{\mathbf{L}}(x, y)=\min (x+y, 1)$.

A t-norm $T$ has zero divisors if there exist $x, y \in] 0,1[$ such that $T(x, y)=0$. Among the above, only $T_{\mathbf{L}}$ has zero divisors. A t-conorm $S$ has zero divisors if there exist $x, y \in] 0,1[$ such that $S(x, y)=1$; sometimes one talks about a t-conorm without unit multipliers instead. Among the above, only $S_{\mathbf{L}}$ has zero divisors.

A fuzzy relation $Q$ on $A$ is a fuzzy subset of $A^{2}$. If $Q(a, b) \in\{0,1\}$ for all $a, b \in A$, then $Q$ is a crisp relation. For a fuzzy relation $Q$ on $A$, we will use the following notations:

$$
\begin{aligned}
& M_{Q}(a, b)=S_{\mathbf{M}}(Q(a, b), Q(b, a)), \\
& m_{Q}(a, b)=T_{\mathbf{M}}(Q(a, b), Q(b, a)) .
\end{aligned}
$$

Given a fuzzy relation $Q$ on $A$, the fuzzy relations $Q^{t}$ and $Q^{c}$ are defined by $Q^{t}(a, b)=Q(b, a)$ and $Q^{c}(a, b)=1-Q(a, b)$, for all $a, b \in A$.

Given a t-norm $T$ and a t-conorm $S$, a fuzzy relation $Q$ on $A$ is called:

(i) reflexive if $Q(a, a)=1$ for all $a \in A$;

(ii) symmetric if $Q(a, b)=Q(b, a)$ for all $a, b \in A$;

(iii) T-asymmetric if $T(Q(a, b), Q(b, a))=0$ for all $a, b \in A$;

(iv) $T$-antisymmetric if $T(Q(a, b), Q(b, a))=0$ for all $a, b \in A, a \neq b$;

(v) T-transitive if $T(Q(a, b), Q(b, c)) \leq Q(a, c)$ for all $a, b, c \in A$;

(vi) $S$-complete if $S(Q(a, b), Q(b, a))=1$ for all $a, b \in A$.

A fuzzy relation is called a T-equivalence if it is reflexive, symmetric and $T$ transitive; it is called a fuzzy partial order with respect to $T$, for brevity a $T$ ordering, if it is reflexive, $T$-antisymmetric and $T$-transitive. However, some authors, such as Höhle and Blanchard ${ }^{12}$ and Bodenhofer ${ }^{13}$ have considered the following definition of a fuzzy partial order. 
Definition 1. A fuzzy relation $R$ on $A$ is called a fuzzy partial order with respect to a t-norm $T$ and a $T$-equivalence $I$ on $A$, for brevity a $T$ - $I$-ordering, if it is:

(i) $I$-reflexive: $I(a, b) \leq R(a, b)$, for all $a, b \in A$;

(ii) $T$-I-antisymmetric: $T(R(a, b), R(b, a)) \leq I(a, b)$, for all $a, b \in A$;

(iii) $T$-transitive.

$T$-orderings are particular cases of $T$ - $I$-orderings and they are obtained when $I$ is the crisp equality $(I=\Delta)$, i.e.,

$$
I(a, b)= \begin{cases}1 & , \text { if } a=b \\ 0 & , \text { if } a \neq b .\end{cases}
$$

Obviously, if $I$ is a $T$-equivalence, then $R$ is a $T$ - $I$-ordering if and only if $R$ is $T$-transitive and for all $a, b \in A$ it holds that

$$
T(R(a, b), R(b, a)) \leq I(a, b) \leq m_{R}(a, b) .
$$

In case the t-norm used is $T_{\mathbf{L}}$, the preceding condition frequently appears in the literature on factorizations of fuzzy weak preference relations (see, for instance, Fodor and Roubens ${ }^{14}$, Llamazares $^{9}$ and De Baets and Fodor $\left.{ }^{10,15}\right)$. For this reason, a natural course of action is the study of fuzzy strict preference relations corresponding to a $T$-equivalence and a $T$ - $I$-ordering. This is realized in the following section.

\section{The results}

In this section, we analyze three cases. The first case considered is the one where the $T$-equivalence $I$ is the crisp equality. In the second case, we focus on t-norms without zero divisors. The final case is that of the important Lukasiewicz t-norm ${ }^{4,5}$. In the latter case, we distinguish between the three most important t-conorms mentioned above, i.e. the Eukasiewicz t-conorm, the maximum and the probabilistic sum.

Before we start our study, we have to make clear what we mean by a fuzzy strict preference relation in the context of $T$ - $I$-orderings. Note that we do not consider a fuzzy incomparability relation, although it is possible that the $T$ - $I$-ordering is not $S$-complete.

Definition 2. Let $T$ be a t-norm, $S$ a t-conorm, $I$ a $T$-equivalence and $R$ a $T$ - $I$ ordering. A fuzzy relation $P$ is called a fuzzy strict preference relation compatible with $(R, I, T, S)$ if $R=P \cup_{S} I, P \cap_{T} I=\emptyset$ and $P \cap_{T} P^{t}=\emptyset$.

Since $S_{\mathrm{M}}$ is the smallest t-conorm, it follows from $R=P \cup_{S} I$ that $P(a, b) \leq$ $R(a, b)$, for all $a, b \in A$. As will be seen further on, additional conditions might have to be imposed upon $I$ and $R$ in order to ensure the existence of a compatible fuzzy strict preference relation. 


\subsection{The crisp equality}

The simplest situation occurs when the $T$-equivalence $I$ is the crisp equality. It then follows from (1) that $T(R(a, b), R(b, a))=0$ for all $a, b \in A, a \neq b$. Therefore, the fuzzy relation $R$ has to be $T$-antisymmetric. Moreover, there is a unique compatible fuzzy strict preference relation. It is very similar to the one in the crisp case: the fuzzy strict preference relation coincides with the fuzzy fuzzy weak preference relation except when both alternatives are equal.

Theorem 1. Let $T$ be a t-norm, $S$ a t-conorm, $I$ the crisp equality and $R$ a $T$ $I$-ordering. A fuzzy relation $P$ is a fuzzy strict preference relation compatible with $(R, I, T, S)$ if and only if for all $a, b \in A$ it holds that

$$
P(a, b)= \begin{cases}R(a, b) & , \text { if } a \neq b, \\ 0 & \text {, if } a=b .\end{cases}
$$

Proof. Suppose first that $P$ is a fuzzy strict preference relation compatible with $(R, I, T, S)$. For any $a, b \in A, a \neq b$, it holds that

$$
P(a, b)=S(P(a, b), 0)=S(P(a, b), I(a, b))=R(a, b) .
$$

Moreover, $P(a, a)=T(P(a, a), 1)=T(P(a, a), I(a, a))=0$.

On the other hand, it is easy to check that the converse statement is true.

In the following theorem we show that this fuzzy strict preference relation is $T$-transitive.

Theorem 2. Let $T$ be a t-norm, $S$ a t-conorm, $I$ the crisp equality and $R$ a $T$ $I$-ordering. The fuzzy strict preference relation compatible with $(R, I, T, S)$ is $T$ transitive.

Proof. Consider $a, b, c \in A$. We distinguish three cases:

(i) If $a, b, c$ are different, then $T(P(a, b), P(b, c)) \leq P(a, c)$ because $R$ is $T$ transitive.

(ii) If $a=b$ or $b=c$, then $T(P(a, b), P(b, c))=0 \leq P(a, c)$.

(iii) If $a \neq b, b \neq c$ and $a=c$, then

$$
T(P(a, b), P(b, c))=T(R(a, b), R(b, a))=0=P,(a, c) .
$$

\subsection{T-norms without zero divisors}

When considering a t-norm without zero divisors, we prove in the following theorem that the fuzzy strict preference relation coincides with the one introduced by Ovchinnikov ${ }^{16}$ in 1981.

Theorem 3. Let $T$ be a t-norm without zero divisors, $S$ a t-conorm, $I$ a $T$ equivalence and $R$ a T-I-ordering. A fuzzy relation $P$ is a fuzzy strict preference 
relation compatible with $(R, I, T, S)$ if and only if for all $a, b \in A$ the fuzzy relation $R$ satisfies

$$
\text { if } m_{R}(a, b)<M_{R}(a, b) \text { then } m_{R}(a, b)=0
$$

and $I$ and $P$ are defined by

$$
\begin{aligned}
& I(a, b)= \begin{cases}R(a, b) & , \text { if } R(a, b)=R(b, a), \\
0 & , \text { if } R(a, b) \neq R(b, a),\end{cases} \\
& P(a, b)= \begin{cases}R(a, b) & , \text { if } R(a, b) \neq R(b, a), \\
0 & , \text { if } R(a, b)=R(b, a) .\end{cases}
\end{aligned}
$$

Before proceeding to the proof, we would like to stress that the existence of a (unique) fuzzy strict preference relation in the above theorem imposes an additional condition on $R$, namely $\min (R(a, b), R(b, a))=0$ if $R(a, b) \neq R(b, a)$, and leaves no choice for $I$.

Proof. Suppose first that $P$ is a fuzzy strict preference relation compatible with $(R, I, T, S)$. Consider $a, b \in A$. Since $T$ has no zero divisors, $I$ is symmetric, $T(P(a, b), I(a, b))=0, T(P(b, a), I(b, a))=0$ and $T(P(a, b), P(b, a))=0$, at most one of $P(a, b), P(b, a)$ and $I(a, b)$ can be strictly positive. We distinguish two cases:

(i) If $R(a, b)=R(b, a)$, then

$$
R(a, b)=S(P(a, b), I(a, b))=S(P(b, a), I(a, b))=R(b, a) .
$$

Consequently, the only possibility is $P(a, b)=P(b, a)=0$ and $I(a, b)=R(a, b)$. Note that the latter is also true if $R(a, b)=R(b, a)=0$.

(ii) If $R(a, b) \neq R(b, a)$ and we suppose that $R(a, b)>R(b, a)$, then

$$
S(P(a, b), I(a, b))>S(P(b, a), I(a, b)) .
$$

The only possibility is $I(a, b)=P(b, a)=R(b, a)=0$ and $P(a, b)=R(a, b)$. Analogously, if we suppose $R(b, a)>R(a, b)$, we obtain $I(a, b)=P(a, b)=$ $R(a, b)=0$ and $P(b, a)=R(b, a)$. This also shows that $R$ satisfies (2).

On the other hand, it is easy to check that the converse statement is true.

Note that the fuzzy strict preference relation $P$ can also be written as (still given that $R$ satisfies (2)):

$$
P(a, b)= \begin{cases}R(a, b) & , \text { if } R(a, b)>R(b, a), \\ 0 & , \text { otherwise. }\end{cases}
$$

This expression facilitates the study of the transitivity of the fuzzy strict preference relation.

Theorem 4. Let $T$ be a t-norm without zero divisors, $S$ a t-conorm, $I$ a $T$ equivalence and $R$ a $T$-I-ordering satisfying (2). The fuzzy strict preference relation $P$ compatible with $(R, I, T, S)$ is T-transitive. 
Proof. Consider $a, b, c \in A$. Since $T$ has absorbing element 0 and $R$ is $T$-transitive, we only need to prove that $T(P(a, b), P(b, c)) \leq P(a, c)$ when $P(a, b)=R(a, b)>0$, $P(b, c)=R(b, c)>0$ and $P(a, c)=0$. In this case, it is also holds that $R(b, a)=$ $R(c, b)=0$ and $R(a, c) \leq R(c, a)$. As $R$ is $T$-transitive, it follows that

$$
T(R(b, c), R(c, a)) \leq R(b, a)=0 .
$$

Since $T$ has no zero divisors and $R(b, c) \neq 0$, it holds that $R(c, a)=0$. The $T$ transitivity of $R$ leads to

$$
T(P(a, b), P(b, c))=T(R(a, b), R(b, c)) \leq R(a, c) \leq R(c, a)=0=P(a, c) .
$$

\subsection{The Eukasiewicz t-norm}

The Lukasiewicz t-norm is one of the most important t-norms in the fuzzy preference modelling framework ${ }^{4,5}$ and will therefore receive ample attention in this section. Concerning the choice of t-conorm, we will consider the three prototypical t-conorms. Before doing so, we consider the general case of an $S$-complete fuzzy weak preference relation, with $S$ a t-conorm without zero divisors. In this case, the crisp case is recovered.

Theorem 5. Let $S$ be a t-conorm without zero divisors, $I$ a $T_{\mathbf{L}}$-equivalence, $R$ an $S$-complete $T_{\mathbf{L}}$-I-ordering. A fuzzy relation $P$ is a fuzzy strict preference relation compatible with $\left(R, I, T_{\mathbf{L}}, S\right)$ if and only if $R, P$ and $I$ are crisp.

Proof. Consider $a, b \in A$. Since $S$ has no zero divisors and $R$ is $S$-complete, it holds that $M_{R}(a, b)=1$. We distinguish two cases:

(i) If $I(a, b)=1$, then also $m_{R}(a, b)=1$ and, since $P(a, b)+I(a, b) \leq 1$ and $P(b, a)+I(a, b) \leq 1$, it follows that $M_{P}(a, b)=0$.

(ii) If $I(a, b)<1$, since $S(P(a, b), I(a, b))=R(a, b)$ and $S(P(b, a), I(a, b))=R(b, a)$, $M_{R}(a, b)=1$ and $S$ has no zero divisors, it holds that $M_{P}(a, b)=1$. Because $P(a, b)+P(b, a) \leq 1$ and $M_{P}(a, b)+I(a, b) \leq 1$, it further holds that $m_{P}(a, b)=$ $I(a, b)=0$ and, consequently, $m_{R}(a, b)=0$.

Combining all the above facts, it is clear that $R, P$ and $I$ are crisp and that $P$ and $I$ are the asymmetric and symmetric part of $R$, respectively.

From here on, we will not suppose the $S$-completeness of the fuzzy weak preference relation and consider the three t-conorms: the Łukasiewicz t-conorm $S_{\mathbf{L}}$, the maximum $S_{\mathbf{M}}$ and the probabilistic sum $S_{\mathbf{P}}$. When the Eukasiewicz t-conorm is considered, we show that $R$ can be obtained from $P$ and $I$ in an additive form. The fuzzy strict preference relation thus obtained is one of the most common ones given in the literature (see, for instance, Fodor and Roubens ${ }^{14}$, De Baets and Fodor ${ }^{10,15}$, Llamazares $^{9}$ and Bodenhofer and Demirci ${ }^{17}$ ). 
Theorem 6. Let $I$ be a $T_{\mathbf{L}}$-equivalence and $R$ a $T_{\mathbf{L}}$-I-ordering. A fuzzy relation $P$ is a fuzzy strict preference relation compatible with $\left(R, I, T_{\mathbf{L}}, S_{\mathbf{L}}\right)$ if and only if for all $a, b \in A$ it holds that

$$
P(a, b)=R(a, b)-I(a, b)
$$

Proof. Suppose first that $P$ is a fuzzy strict preference relation compatible with $\left(R, I, T_{\mathbf{L}}, S_{\mathbf{L}}\right)$. Consider $a, b \in A$. Since $P(a, b)+I(a, b) \leq 1$ and $R(a, b)=$ $\min (P(a, b)+I(a, b), 1)$, it holds that $R(a, b)=P(a, b)+I(a, b)$, i.e., $P(a, b)=$ $R(a, b)-I(a, b)$.

Assume now that $P$ is defined by $P(a, b)=R(a, b)-I(a, b)$, then we have to show that it is compatible with $\left(R, I, T_{\mathbf{L}}, S_{\mathbf{L}}\right)$. Since $P(a, b)+I(a, b)=R(a, b) \leq 1$, for all $a, b \in A$, it trivially holds that $R=P \cup_{S_{\mathrm{L}}} I$ and $P \cap_{T_{\mathrm{L}}} I=\emptyset$. It remains to prove that $P \cap_{T_{\mathbf{L}}} P^{t}=\emptyset$, i.e., for all $a, b \in A$ it holds that $P(a, b)+P(b, a) \leq 1$. Since $R$ is a $T_{\mathbf{L}}-I$-ordering, it holds that $R(a, b)+R(b, a)-1 \leq I(a, b)$. Hence, $R(a, b)+R(b, a)-1 \leq 2 I(a, b)$, or equivalently,

$$
R(a, b)-I(a, b)+R(b, a)-I(b, a) \leq 1,
$$

i.e., $P(a, b)+P(b, a) \leq 1$.

Dasgupta and Deb ${ }^{18}$ have proven that if $R$ is $S_{\mathbf{L}}$-complete and $I(a, b)=$ $T_{\mathbf{L}}(R(a, b), R(b, a))$ or $I(a, b)=m_{R}(a, b)$, then $P$ is $T_{\mathbf{L}}$-transitive. As the following example shows, if $I$ is different from the previous cases it is possible that $P$ is not $T_{\mathbf{L}}$-transitive, even when $R$ is $S_{\mathbf{L}}$-complete. Extensive results on the propagation of transitivity can be found in Díaz et al. ${ }^{19,20,21,22,23}$.

Example 1. Let $A=\{a, b, c\}$ and consider the following fuzzy relations in matrix representation:

$$
R=\left(\begin{array}{ccc}
1 & 0.8 & 0.6 \\
0.4 & 1 & 0.8 \\
0.6 & 0.4 & 1
\end{array}\right), \quad I=\left(\begin{array}{ccc}
1 & 0.2 & 0.6 \\
0.2 & 1 & 0.3 \\
0.6 & 0.3 & 1
\end{array}\right), \quad P=\left(\begin{array}{ccc}
0 & 0.6 & 0 \\
0.2 & 0 & 0.5 \\
0 & 0.1 & 0
\end{array}\right)
$$

It is easy to verify that $I$ is a $T_{\mathbf{L}}$-equivalence, $R$ is a $S_{\mathbf{L}}$-complete $T_{\mathbf{L}}$ - $I$-ordering and $P$ is a fuzzy strict preference relation compatible with $\left(R, I, T_{\mathbf{L}}, S_{\mathbf{L}}\right)$. However, $P$ is not $T_{\mathbf{L}}$-transitive since $P(a, b)+P(b, c)-1>P(a, c)$.

The second t-conorm we consider is $S_{\mathrm{M}}$. In this case, the fuzzy strict preference relation is not defined in a unique way.

Theorem 7. Let $I$ be a $T_{\mathbf{L}}$-equivalence and $R$ a $T_{\mathbf{L}}$-I-ordering. A fuzzy binary relation $P$ is a fuzzy strict preference relation compatible with $\left(R, I, T_{\mathbf{L}}, S_{\mathbf{M}}\right)$ if and only if for all $a, b \in A$ the fuzzy relations $I$ and $R$ satisfy

$$
\text { if } I(a, b)<M_{R}(a, b) \text { then } R(a, b)+R(b, a) \leq 1
$$

and $P$ is defined by 
(i) if $I(a, b)<R(a, b)$, then $P(a, b)=R(a, b)$;

(ii) if $I(a, b)=R(a, b)$, then $P(a, b) \leq \min (R(a, b), 1-R(a, b))$.

Proof. Suppose first that $P$ is a fuzzy strict preference relation compatible with $\left(R, I, T_{\mathbf{L}}, S_{\mathbf{M}}\right)$. Since $R=P \cup_{S_{\mathbf{M}}} I, P \cap_{T_{\mathbf{L}}} I=\emptyset$ and $P \cap_{T_{\mathbf{L}}} P^{t}=\emptyset$, for all $a, b \in A$ it holds that:

$$
\begin{aligned}
& R(a, b)=\max (P(a, b), I(a, b)), \\
& R(b, a)=\max (P(b, a), I(a, b)), \\
& P(a, b)+I(a, b) \leq 1, \\
& P(a, b)+P(b, a) \leq 1 .
\end{aligned}
$$

We distinguish two cases:

(i) If $R(a, b)=R(b, a)$, then in view of (4) we consider again two cases:

(a) If $I(a, b)=R(a, b)$, then (4) and (6) imply that $P(a, b) \leq R(a, b)$ and $P(a, b) \leq 1-R(a, b)$, i.e.

$$
P(a, b) \leq \min (R(a, b), 1-R(a, b)) .
$$

(b) If $I(a, b)<R(a, b)$, then (4) and (5) imply that $P(a, b)=P(b, a)=R(a, b)$. In view of (7), this is only possible if $R(a, b)+R(b, a) \leq 1$, i.e. (3) should hold.

(ii) If $R(a, b) \neq R(b, a)$, suppose, for instance, that $R(b, a)<R(a, b)$. From (5) we distinguish two cases:

(a) If $I(a, b)=R(b, a)<R(a, b)$, it follows from (4) that $P(a, b)=R(a, b)$. In view of (6), this is only possible if $R(a, b)+R(b, a) \leq 1$, i.e. (3) should hold.

(b) If $I(a, b)<R(b, a)<R(a, b)$, it follows from (4) and (5) imply that $P(a, b)=$ $R(a, b)$ and $P(b, a)=R(b, a)$. Condition (7) again imposes that $R(a, b)+$ $R(b, a) \leq 1$.

The case $R(a, b)<R(b, a)$ is totally similar.

Combining the above cases, the proof is complete.

On the other hand, it is easy to check that the converse statement is true.

Note that the above theorem leads to a whole class of fuzzy strict preference relations compatible with $\left(R, I, T_{\mathbf{L}}, S_{\mathbf{M}}\right)$. It is simple to determine the smallest and greatest members of this class.

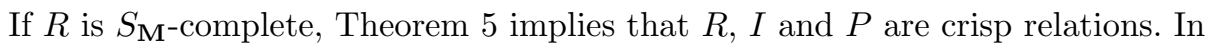
that case, $P$ is trivially $T_{\mathbf{L}}$-transitive if $R$ is $T_{\mathbf{L}}$-transitive. However, as the following example shows, if $R$ is not $S_{\mathrm{M}^{-}}$complete, then it is possible that $P$ is not $T_{\mathbf{L}^{-}}$ transitive. 
Example 2. Let $A=\{a, b, c\}$ and consider the following fuzzy relations in matrix representation:

$$
R=\left(\begin{array}{ccc}
1 & 0.6 & 0.6 \\
0.2 & 1 & 0.5 \\
0.6 & 0.3 & 1
\end{array}\right), \quad I=\left(\begin{array}{ccc}
1 & 0.2 & 0.6 \\
0.2 & 1 & 0.3 \\
0.6 & 0.3 & 1
\end{array}\right), \quad P=\left(\begin{array}{ccc}
0 & 0.6 & 0 \\
0.2 & 0 & 0.5 \\
0 & 0.1 & 0
\end{array}\right)
$$

It is easy to check that $I$ is a $T_{\mathbf{L}}$-equivalence, $R$ is a $T_{\mathbf{L}}$-I-ordering and $P$ is a fuzzy strict preference relation compatible with $\left(R, I, T_{\mathbf{L}}, S_{\mathbf{M}}\right) . P$ is the same fuzzy relation as in Example 1. Consequently, it is not $T_{\mathbf{L}}$-transitive.

Finally, we consider the t-conorm $S_{\mathbf{P}}$. In this case, to our knowledge, the unique fuzzy strict preference relation obtained has not been mentioned before in the literature. We introduce the following notation: $Z(a, b)=\sqrt{M_{R}(a, b)-3 / 4}$.

Theorem 8. Let $I$ be a $T_{\mathbf{L}}$-equivalence and $R$ a $T_{\mathbf{L}}$-I-ordering. A fuzzy relation $P$ is a fuzzy strict preference relation compatible with $\left(R, I, T_{\mathbf{L}}, S_{\mathbf{P}}\right)$ if and only if for all $a, b \in A$ the fuzzy relations $I$ and $R$ satisfy

$$
\text { if } M_{R}(a, b)>3 / 4 \text { then } I(a, b) \leq 1 / 2-Z(a, b) \text { or } 1 / 2+Z(a, b) \leq I(a, b)
$$

and $P$ is defined by

$$
P(a, b)= \begin{cases}\frac{R(a, b)-I(a, b)}{1-I(a, b)} & , \text { if } I(a, b)<1, \\ 0 & , \text { if } I(a, b)=1 .\end{cases}
$$

Proof. Suppose first that $P$ is a strict preference relation compatible with $\left(R, I, T_{\mathbf{L}}, S_{\mathbf{P}}\right)$. Since $R=P \cup_{S_{\mathbf{P}}} I$ and $P \cap_{T_{\mathbf{L}}} I=\emptyset$, for all $a, b \in A$ it holds that:

$$
\begin{aligned}
& R(a, b)=P(a, b)+I(a, b)-P(a, b) I(a, b), \\
& P(a, b)+I(a, b) \leq 1 .
\end{aligned}
$$

If $I(a, b)<1$, then it follows from $(9)$ that

$$
P(a, b)=\frac{R(a, b)-I(a, b)}{1-I(a, b)} .
$$

On the other hand, if $I(a, b)=1$, then (10) implies that $P(a, b)=0$. Moreover, in that case, $M_{R}(a, b)=1$ and (8) is trivially fulfilled.

Therefore, suppose that $I(a, b)<1$, then substitution of $P(a, b)$ in (10) yields

$$
\frac{R(a, b)-I(a, b)}{1-I(a, b)}+I(a, b) \leq 1
$$

or equivalently,

$$
R(a, b) \leq(I(a, b))^{2}-I(a, b)+1 .
$$

Since $x^{2}-x+1 \geq 3 / 4$ for all $x \in[0,1]$, we have that (12) is always fulfilled if $R(a, b) \leq$ $3 / 4$. On the other hand, if $R(a, b)>3 / 4$, then $(I(a, b))^{2}-I(a, b)+(1-R(a, b)) \geq 0$ 
if and only if $I(a, b) \leq 1 / 2-\sqrt{R(a, b)-3 / 4}$ or $I(a, b) \geq 1 / 2+\sqrt{R(a, b)-3 / 4}$. The same conditions obviously hold for $R(b, a)$. Hence, if $M_{R}(a, b)>3 / 4$ then (12) is satisfied if and only if $I(a, b) \leq 1 / 2-Z(a, b)$ or $I(a, b) \geq 1 / 2+Z(a, b)$.

On the other hand, it is easy to check that the converse statement is true.

If $R$ is $S_{\mathbf{P}}$-complete, Theorem 5 implies that $R, I$ and $P$ are crisp relations. In that case, $P$ is trivially $T_{\mathbf{L}}$-transitive if $R$ is $T_{\mathbf{L}}$-transitive. However, as the following

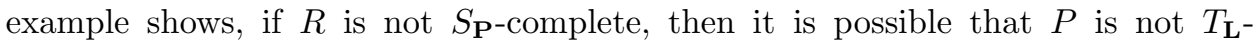
transitive.

Example 3. Let $A=\{a, b, c\}$ and consider the following fuzzy relations in matrix representation:

$$
R=\left(\begin{array}{ccc}
1 & 0.68 & 0.6 \\
0.36 & 1 & 0.65 \\
0.6 & 0.37 & 1
\end{array}\right), \quad I=\left(\begin{array}{ccc}
1 & 0.2 & 0.6 \\
0.2 & 1 & 0.3 \\
0.6 & 0.3 & 1
\end{array}\right), \quad P=\left(\begin{array}{ccc}
0 & 0.6 & 0 \\
0.2 & 0 & 0.5 \\
0 & 0.1 & 0
\end{array}\right)
$$

It is easy to check that $I$ is a $T_{\mathbf{L}}$-equivalence, $R$ is a $T_{\mathbf{L}}-I$-ordering and $P$ is a strict preference relation compatible with $\left(R, I, T_{\mathbf{L}}, S_{\mathbf{P}}\right) . P$ is the same fuzzy relation as in Examples 1 and 2 . Consequently, it is not $T_{\mathbf{L}}$-transitive.

\section{Concluding Remarks}

The classical theory of preference modelling provides a unique way to obtain a strict preference relation from a weak preference relation. However, in the fuzzy framework, different fuzzy strict preference relations can be obtained from the same fuzzy weak preference relation, simply because there exist different ways for generalizing crisp properties. In this paper, we have investigated the compatibility of fuzzy strict preference relations with fuzzy partial orders, a particular class of fuzzy weak preference relations.

\section{Acknowledgements}

This work is partially financed by the Junta de Castilla y León (Project VA002B08), the Spanish Ministry of Education and Science (Project SEJ2006-04267) and ERDF.

\section{References}

1. M. Roubens and P. Vincke, Preference Modeling (Lecture Notes in Economics and Mathematical Systems 250, Springer, Berlin, 1985).

2. B. De Baets and B. Van de Walle, "Minimal definitions of classical and fuzzy preference structures", Proc. of the Annual Meeting of the North American Fuzzy Information Processing Society, Syracuse, Sept. 1997, pp. 299-304.

3. P. Perny and M. Roubens, "Fuzzy preference modeling", in Fuzzy Sets in Decision Analysis, Operations Research and Statistics, ed. R. Słowiński (Kluwer Academic Publishers, Dordrecht, 1998) pp. 3-30. 
4. B. Van de Walle, B. De Baets and E. Kerre, "A plea for the use of Łukasiewicz triplets in the definition of fuzzy preference structures. Part 1: General argumentation", Fuzzy Sets and Systems 97 (1998) 349-359.

5. B. De Baets, B. Van de Walle and E. Kerre, "A plea for the use of Łukasiewicz triplets in the definition of fuzzy preference structures. Part 2: The identity case", Fuzzy Sets and Systems 99 (1998) 303-310.

6. B. Van de Walle, B. De Baets and E. Kerre, "Characterizable fuzzy preference structures", in Annals of Operations Research 80, Special Issue Preference Modeling, eds. D. Bouyssou and P. Vincke (Baltzer Science Publishers, Bussum, 1998) pp. 105-136.

7. A. Bufardi, "On the construction of fuzzy preference structures", Journal of MultiCriteria Decision Analysis 7 (1998) 169-175.

8. A. Bufardi, "On the fuzzification of the classical definition of preference structure", Fuzzy Sets and Systems 104 (1999) 323-332.

9. B. Llamazares, "Characterization of fuzzy preference structures through Łukasiewicz triplets", Fuzzy Sets and Systems 136 (2003) 217-235.

10. B. De Baets and J. Fodor, "Additive fuzzy preference structures: the next generation", in: Principles of Fuzzy Preference Modelling and Decision Making, eds. B. De Baets, J. Fodor (Academia Press, 2003) pp. 15-25.

11. L.A. Fono, H. Gwet and S. Fotso, "On strict lower and upper sections of weakly complete fuzzy pre-orders based on co-implication", Fuzzy Sets and Systems 159 (2008) 2240-2255.

12. U. Höhle and N. Blanchard, "Partial ordering in L-underdeterminate sets", Information Sciences 35 (1985) 133-144.

13. U. Bodenhofer, "A similarity-based generalization of fuzzy orderings preserving the classical axioms", International Journal of Uncertainty, Fuzziness and KnowledgeBased Systems 8 (2000) 593-610.

14. J. Fodor and M. Roubens, Fuzzy Preference Modelling and Multicriteria Decision Support (Kluwer Academic Publishers, Dordrecht, 1994).

15. B. De Baets and J. Fodor, "Fuzzy preference modelling: Fundamentals and recent advances", in Fuzzy Sets and Their Extensions: Representation, Aggregation and Models, eds. H. Bustince, F. Herrera and J. Montero (Springer-Verlag, Berlin, 2008) pp. 207-217.

16. S. Ovchinnikov, "Structure of fuzzy binary relations", Fuzzy Sets and Systems 6 (1981) 169-195.

17. U. Bodenhofer and M. Demirci, "Strict fuzzy orderings with a given context of similarity", International Journal of Uncertainty, Fuzziness and Knowledge-Based Systems 16 (2008) 147-178.

18. M. Dasgupta and R. Deb, "Factoring fuzzy transitivity", Fuzzy Sets and Systems 118 (2001) 489-502.

19. S. Díaz, S. Montes and B. De Baets, "Transitive decomposition of fuzzy preference relations: the case of nilpotent minimum", Kybernetika 40 (2004) 71-88.

20. S. Díaz, B. De Baets and S. Montes, "Additive decomposition of fuzzy pre-orders", Fuzzy Sets and Systems 158 (2007) 830-842.

21. S. Díaz, S. Montes and B. De Baets, "Transitivity bounds in additive fuzzy preference structures", IEEE Trans. Fuzzy Systems 15 (2007) 275-286.

22. S. Díaz, B. De Baets and S. Montes, "On the compositional characterization of complete fuzzy pre-orders", Fuzzy Sets and Systems 159 (2008) 2221-2239.

23. S. Díaz, B. De Baets and S. Montes, "General results on the decomposition of transitive fuzzy relations", Fuzzy Optimization and Decision Making, in press. 